\title{
A clinical decision aid was accurate for predicting survival to hospital discharge after in-hospital cardiac resuscitation
}

\author{
van Walraven C, Forster AJ, Parish DC, et al. Validation of a clinical decision aid to discontinue in-hospital cardiac arrest \\ resuscitations. JAMA 2001 Mar 28;285:1602-6.
}

\section{QUESTION: How accurate is a clinical decision aid for identifying patients having in-hospital cardiac resuscitation who would survive to hospital discharge?}

\section{Design}

Analysis of patient records from a large registry of in-hospital resuscitations to validate a previously derived clinical decision aid.

\section{Setting}

A 550 bed community teaching hospital in Macon, Georgia, USA.

\section{Patients}

Patients $\geq 16$ years of age who had an in-hospital resuscitation attempt for cardiac arrest with an initial rhythm of either pulseless ventricular tachycardia, ventricular fibrillation, pulseless electrical activity, or asystole. Patients in the emergency department were included only if they had an arrest after they arrived in the department. Patients were excluded if resuscitation was done in the operating room, they received no chest compression, the time to initial chest compression was $>15$ minutes, or the information needed by the decision aid was missing.

\section{Description of prediction guide}

Patients were predicted to have a chance of being discharged from the hospital if their arrest was witnessed, their initial cardiac rhythm was either ventricular tachycardia or ventricular fibrillation, or they attained a pulse lasting $\geqslant 2$ minutes during the first 10 minutes of chest compression. Patients were classified as having a "witnessed arrest" if they were directly seen to lose spontaneous circulation or if they arrested while on a cardiac monitor, in the intensive or coronary care unit, or in the catheterisation laboratory.

\section{Main outcome measure}

Survival to hospital discharge.

Sources of funding:

Physicians'Services

Foundation Arthur

Bond Scholarship and

Ontario Ministry of

Health.

For correspondence: Dr C van Walraven, Department of Medicine, University of Ottawa, F-660, 1053 Carling Avenue, Ottawa, Ontario $K 1 Y$ 4E9, Canada. Fax +1 6137615351.

\section{Main results}

Data from 2181 in-hospital resuscitation attempts in 1884 patients (mean age $65 \mathrm{y}, 53 \%$ men) were used to validate the decision aid. $69 \%$ of arrests had a cardiorespiratory cause; $79 \%$ were witnessed; and $96 \%$, had chest compression begun within 5 minutes of arrest. For 327 resuscitations $(15 \%)$, the patient survived to discharge from the hospital; for 324 of these resuscitations, the patient was predicted to have a chance of survival to hospital discharge. The sensitivity, specificity, and likelihood ratios for the clinical decision aid are shown in the table. Of 269 resuscitations for which the decision aid predicted no chance of surviving to hospital discharge, only 3 patients were discharged from the hospital, and none were able to live independently after discharge.

\section{Conclusion}

A clinical decision aid was accurate for identifying patients having in-hospital cardiac resuscitation who could survive to hospital discharge.

Test characteristics of a clinical decision aid for predicting survival to hospital discharge after in-hospital cardiac resuscitation *

\begin{tabular}{llll} 
Sensitivity $(95 \% \mathrm{Cl})$ & Specificity $(\mathrm{Cl})$ & +LR & -LR \\
\hline $99.1 \%(97.1$ to 99.8$)$ & $14.4 \%(12.4$ to 16.0$)$ & 1.16 & 0.06 \\
\hline
\end{tabular}

*Diagnostic terms defined in glossary; LRs calculated from data in article.

\section{COMMENTARY}

The decision aid developed by van Walraven et al was simple and used intra-arrest factors that are easily obtainable during an ongoing resuscitation attempt. The aid successfully identified patients with an exceedingly low likelihood of surviving the hospital stay $(1.1 \%, 95 \%$ CI $0.3 \%$ to $3.5 \%)$ : that is, patients in whom the arrest was not witnessed, the initial rhythm was not ventricular tachycardia or ventricular fibrillation, and a pulse was not restored during the first $10 \mathrm{~min}$ utes of chest compression. The predictive values were similar in the derivation and validation groups despite differences in age, location of arrest, and survival outcomes, which suggest that the decision aid is robust and potentially applicable to other hospital settings.

This study implies that discontinuing ongoing resuscitation efforts when poor hospital survival is predicted with this decision aid may be reasonable. However, temporary return of life might be a desirable outcome in some patients Approximately $20 \%$ of the non-survivors were initially admitted to the intensive care unit, and 10\% lived for $>24$ hours (mean $8.5 \mathrm{~d}$ ). van Walraven et al highlight that $75 \%$ of these patients never regained consciousness; however, the retrospective nature of the study precludes a more detailed analysis. Further work is needed to assess the characteristics of this short-term outcome and its significance to patients and their surrogate decision-makers.

Finally, the decision aid was developed by using conventional closed-chest resuscitation techniques. Recent advances in resuscitation research could yield more potent interventions for which new decision aid rules might be required.

Raul J Gazmuri, MD, PhD North Chicago VA Medical Center North Chicago, Illinois, USA 